\title{
Primary monosymptomatic nocturnal enuresis: an etiological study
}

\author{
Wafik Said Bahnasy * (D), Yasser Abo Elfotoh El-Heneedy, Ehab Ahmed Shawky El-Seidy, \\ Ibrahim Salah Eldeen Ibrahim, Mohammad Abdel-Hakeem Seleem and Amira Youssef Ahmed
}

\begin{abstract}
Background: Nocturnal enuresis (NE) is a very common chronic pediatric problem with bad psychological consequences.

Methods: Forty primary monosymptomatic nocturnal enuresis (MNE) children and 20 healthy controls were recruited in the study and subjected to history taking, neurological and urological examinations, and psychological assessment using the Arabic-translated and validated version of child behavior checklist, sleep architecture studying through onenight polysomnography (PSG), and vasopressin levels determination both diurnal and nocturnal.

Results: Enuretic children had positive family history of NE in $42.5 \%$, inverted vasopressin circadian rhythm in $52.5 \%$ and PSG changes in the form of increased N3 deep sleep \% of total sleep time (TST), sleep stage transition index (SSTI), periodic limb movement index (PLMI), and snore index. Enuretic children PSG showed decreased deep sleep latency, N1\% of TST, N2\% of TST, and REM \% Of TST. The child behavior checklist showed higher anxious depressed symptoms, social problem, attention problems, and internalizing problems in enuretic children than control subjects.

Conclusions: MNE is a heterogeneous disorder with multiple factors interplay in its pathogenesis. So, the management must be tailored patient by patient according to the dominating etiology.
\end{abstract}

Keywords: Monosymptomatic nocturnal enuresis, Child behavior checklist, Vasopressin, Polysomnography

\section{Background}

Nocturnal enuresis (NE) is defined as a complete or nearly complete micturition in bed during sleep more than two times a week for at least three consecutive months in a healthy child above the age of 5 years (Austin et al. 2014). Following allergy, NE is the second most common chronic pediatric problem affecting $10-15 \%$ of children by the age of 5 years (Butler \& Heron 2008). Spontaneous remission is usual and the number of affected children reduces year by year to reach $1 \%$ at adulthood (Mota et al. 2005). Bedwetting has a deep impact on social, scholastic, and emotional well-being of children during an important period of psychosocial development (Joinson et al. 2007).

Nocturnal enuresis may be functional or organic due to neurological or urological diseases. The organic causes of NE constitute less than $5 \%$ of cases (Joinson et al. 2007). Functional enuresis may be primary with no

\footnotetext{
* Correspondence: wafiq.elbahnasi@med.tanta.edu.eg

Departments of Neuropsychiatry, Chest and Clinical Pathology, Faculty of Medicine, Tanta University, Tanta 31511, Egypt
}

prior period of sustained dryness or secondary with recurrence of nocturnal bed wetting after more than 6 months of dryness. Secondary NE is usually precipitated by upper airway obstruction, urinary tract infection, chronic constipation, ADHD, conduct disorder, specific phobia, generalized anxiety, or depression (Khedr et al. 2015).

Primary NE may be either monosymptomatic nocturnal enuresis (MNE) with normal daytime voiding patterns or non-monosymptomatic NE caused by overactive bladder and presented by daytime wetting, urinary frequency, urgency, hesitancy and interrupted stream with variable-sized wet patches, repeated lower urinary or genital pain, and awakening after wetting (Khedr et al. 2015; Telli et al. 2015).

Primary MNE is a heterogenous disorder results from the interplay of different factors, the predominant one may vary from patient to another which necessitate tailoring of management protocol case by case. The presence of more than one affected family member (history 
of affected parent or siblings) is common pointing to the role of genetic predisposition in MNE. Another possible etiology of MNE is nocturnal polyuria with decreased arousal from full bladder during sleep due to brainstem enuresis inhibiting reflex maturational delay. Psychological factors are also possible causes which if not properly treated results in resistance of many cases to treatments (Baeyens et al. 2007; Raheem et al. 2013).

\section{Aim of the work}

To study the possible primary monosymptomatic nocturnal enuresis etiologies and their relation to the disease severity

\section{Methods}

The present study was conducted on 40 primary MNE children (group I), aged 6-18 years recruited from the outpatient clinics of the Neuropsychiatry Department, Tanta University Hospitals, started from 25 April 2016 till the end of November 2016. Twenty healthy control subjects matching the enuretic subjects' age and sex were also included (group II).

Exclusion criteria included children with non-MNE, secondary NE, mental disorders, and psychiatric problems. NE cases due to organic neurological or urological causes were also excluded which were attained by neurological and urological examinations, urine analysis, and abdominal ultrasound.

The study protocol was approved by the local ethics committee (The Research Ethics Committee and Quality Assurance Unit, Faculty of Medicine, Tanta University). Participation was voluntary, and all participants' parents received detailed information concerning the aims of this research work and possible risks of participation, and an informed consent was obtained from every case prior to the commencement of the study.

History taking was done to all subjects with special stress on frequency of NE, family history of NE, and previous treatment trials.

Patients and control subjects were subjected to one-night polysomnogram (PSG) using a Somon Medics Gmbh (Am SonnenstuhL63, D-97236 Rander Sacker, Germany, type: SOMNO screenTMplus, SN: 4259, kw45: 2014). Each PSG included EEG channels montages (O1/A2, C3/A2, C4/A1, and O2/A1), electrooculography (LOC-A1/A2 and ROC-A 1/A2), surface tibial and submental EMG, and modified V2 lead ECG; nasal and oral signals were recorded by thermal airflow sensors, tracheal sounds by microphone, and the chest and abdominal effort was measured by dual thoracoabdominal RIP (respiratory inductance plethysmography) belts of children size. The PSG parameters were scored according to The American Academy of Sleep Medicine Manual for the Scoring of Sleep and Associated Events: Rules, Terminology and Technical Specifications, (version
2.4), 2017 (Berry et al. 2017), that included the sleep latency (SL) which refers to the length of time taken in transition from wakefulness to sleep, sleep efficiency (SE) which is the total sleep time (TST) divided by the total in bedtime, and sleep stage transition index (SSTI) which is the number of transition between various sleep stages per hour.

Recruited children were also subjected to evaluation of vasopressin level both diurnal (at 9-11 am) and nocturnal (at 9-11 pm). There were no restrictions in water intake of all subjects in the days prior to sample collection but were advised to stop taking any medications in the previous $24 \mathrm{~h}$. Seven milliliters of venous blood was collected from all subjects for biochemical analysis which was divided into $5 \mathrm{ml}$ for obtaining serum samples which were recovered and immediately aliquotted and frozen at $-70{ }^{\circ} \mathrm{C}$ until use. Antidiuretic hormone/vasopressin/arginine vasopressin (ADH/VP/ AVP) was quantified by the commercially available Quantikine Enzyme Linked Immunosorbent Assay (ELISA) methodology according to the manufacturer's instructions (SunRed Assay, Catalog Number 201-12-1049, PELOBIOTECH GmbH-Am Klopferspitz 19-82152 Planegg, Germany). Color intensity was measured by a standard ELISA Reader. All samples and Quantikine kit standards were measured in duplicates for construction of standard curves with normal vasopressin level 2-400 picogram $/ \mathrm{ml}$.

An Arabic-translated and validated version of child behavior checklist (CBCL) was completed by a parent and scored using a computerized scoring software system (Assessment Data Manager-version 9.1). CBCL yields four global T-scores: competence, internalizing behavior problems, externalizing behavior problems, and total problem behaviors. Three subscales contribute to the competence score: activities, social adaptation, and school. Eight subscales contribute to the behavioral syndrome scales: withdrawn, somatic complaints, anxious/ depressed, social problems, thought problems, attention problems, delinquent behaviors, and aggression (Achenbach and Dumenci 2001; EL Koumi et al. 2012).

Statistical analysis was conducted using SPSS version 19 (Statistical Package for Social Studies) created by IBM, Chicago, IL, USA. For numerical values, the mean \pm standard deviation was used and analyzed by the unpaired Student $t$ test. Correlation analysis was performed using Pearson's correlation test. $P$ value ${ }^{<} 0.05$ considered statistically significant.

\section{Results}

The study included $40 \mathrm{MNE}$ child, 24 males $(60 \%)$ and 16 females $(40 \%)$. Their ages were 6.5-17.2 (11.6 \pm 3.1$)$ years, and the bedwetting frequencies were 3-7/week $(4.75 \pm 1.3)$. Seventeen patients $(42.5 \%)$ had positive 
family history of NE (5 had history of an affected parent and 12 had an affected sibling).

Eighteen NE children had history of desmopressin treatment trial; 5 had nearly complete response for more than 3 months but response was drug dependent and rapid relapse of bedwetting occurred on drug stoppage, 4 had partial response with decreased bedwetting frequency by about $50 \%$, and 9 had sluggish or no response. There was no significant difference in the incidence of spina bifida occulta (SBO) among patients and control subjects (22.5 versus $20 \%$ respectively). Six enuretic SBO children were in those with sluggish response to desmopressin and 3 had no previous treatment trials.

The present study showed mildly significant decreased diurnal and nocturnal VP levels in enuretics than healthy control subjects $(202.8 \pm 71.8$ and $194 \pm 73.3$ versus 243.8 \pm 58.6 and $237.2 \pm 56.2$, respectively) with $p=0.031$ and 0.033 . Twenty-one enuretic cases $(52.5 \%)$ showed inverted VP circadian rhythm with nocturnal VP level lower than diurnal versus only 2 controls (10\%). Vasopressin noctur$\mathrm{nal} /$ diurnal difference was significantly lower in enuretic children than control subjects $(-8.88 \pm 37.02$ versus 30.22 \pm 18.29 and $p<0.0001$ ) (Table 1 ).

Regarding $\mathrm{CBCL}$, there were significant increase in the anxious depressed symptoms, social problem, attention problems, and internalizing problems in enuretic children than control subjects $(57.5 \pm 8.5,60.3 \pm 7.1,57.4 \pm$ 7.2 , and $55.6 \pm 10.9$ versus $51.7 \pm 2.3,54.1 \pm 4.5,52.9 \pm$ 3.2 , and $45.8 \pm 8.6$ ) with $p$ values $=0.004,0.0011,0.012$, and 0.0009 , respectively (Table 1 ). Somatic symptoms, thought problems, physical activities, and sluggish cognitive tempo showed non-significant differences between both groups.

Polysomnographic findings showed non-significant differences between MNE children and control subjects regarding TST, SE, and apnea hypopnea index (AHI). The study showed significant decrease in deep sleep latency, N1\% (non-REM 1) of TST, N2\% of TST, and REM \% of
TST in enuretic children than controls $(19.5 \pm 3.6,4.6 \pm$ $1.6,28.7 \pm 11.3$, and $12.8 \pm 6$ versus $25.9 \pm 3.7,5.5 \pm$ $1.4,52.3 \pm 3.5$, and $22.1 \pm 3.8$ ) with $p<0.0001,0.027$, $<0.0001$, and $<0.0001$, respectively. On the other hand, there were increases in each of N3\% of TST, sleep stage transition index (SSTI), periodic limb movement index (PLMI), and snore index in MNE children than control subjects $(54 \pm 18.4,19.5 \pm 6.4,6.7 \pm 2.6$, and $15.2 \pm$ 15.9 versus $20.1 \pm 4,9.7 \pm 2.3,4.77 \pm 0.8$, and $6.5 \pm 2.9$ ) with $p<0.0001,<0.0001,0.002$, and 0.018 , respectively. The study also showed non-significant differences between both groups regarding stages 1, 2, and REM latencies (Table 2 and Figs. 1 and 2).

The frequency of NE was higher among children with positive family history of NE especially those having SBO. It was positively correlated with N3\% of TST, SSTI, and snore index. On the other hand, the frequency was negatively correlated with child's age, N1, N2, and REM $\%$ of TST and nocturnal diurnal vasopressin difference but it had no correlation with PLMs index and the studied psychological parameters of CBCL (Table 3).

Nocturnal diurnal vasopressin difference was positively correlated with N1, N2, and REM \% of TST but negatively correlated with N3\% of TST, SSTI, and snore index (Table 3).

\section{Discussion}

Nocturnal enuresis is defined as involuntary voiding of urine during sleep beyond the age at which bladder control is normally obtained. The primary monosymptomatic variant of NE is a heterogenous disorder in which multiple factors work together to result in its development in genetically predisposed subjects (von Gontard et al. 2011). This work studied the possible etiological factors of MNE and parameters that increase its severity or may results in inappropriate response to treatments. The study showed that MNE is more common in male children with positive family history of NE pointing to the possible hereditary factor in its pathogenesis. These results are in accordance with

Table 1 Vasopressin level and child behavior checklist results among studied enuretic children (group I) and healthy control subjects (group II)

\begin{tabular}{|c|c|c|c|c|c|c|c|}
\hline & & \multicolumn{2}{|l|}{ Group I } & \multicolumn{2}{|l|}{ Group II } & \multicolumn{2}{|l|}{$t$ test } \\
\hline & & Range & Mean \pm SD & Range & Mean \pm SD & $t$ value & $p$ value \\
\hline \multirow[t]{3}{*}{ VP } & Diurnal & $78.3-387.2$ & $202.8 \pm 71.8$ & $65.8-318.5$ & $243.8 \pm 58.6$ & 2.20 & $0.031^{*}$ \\
\hline & Nocturnal & $54.7-320.1$ & $194 \pm 73.3$ & $96.4-334.9$ & $237.2 \pm 56.2$ & 2.18 & $0.033^{*}$ \\
\hline & ND VP differences & $-93.1-55.2$ & $-8.88 \pm 37$ & $-30.9-66.7$ & $30.22 \pm 18.3$ & 4.44 & $<0.0001^{*}$ \\
\hline \multirow[t]{4}{*}{$\mathrm{CBCL}$} & ADS & $50-86$ & $57.5 \pm 8.5$ & $50-57$ & $51.7 \pm 2.3$ & 2.96 & $0.004^{*}$ \\
\hline & Social problems & $50-78$ & $60.3 \pm 7.1$ & $50-66$ & $54.1 \pm 4.5$ & 3.43 & $0.0011^{*}$ \\
\hline & Attention problems & $50-83$ & $57.4 \pm 7.2$ & $50-64$ & $52.9 \pm 3.2$ & 2.56 & $0.012^{*}$ \\
\hline & Internalizing problems & $34-82$ & $55.6 \pm 10.9$ & $33-59$ & $45.8 \pm 8.6$ & 3.505 & $0.0009^{*}$ \\
\hline
\end{tabular}

$A D S$ anxious depressed symptoms, $C B C L$ child behavior checklist, $N D$ nocturnal diurnal, $V P$ vasopressin 
Table 2 Polysomnographic sleep parameters among studied enuretic children (group I) and healthy control subjects (group II)

\begin{tabular}{|c|c|c|c|c|c|c|}
\hline & \multicolumn{2}{|l|}{ Group I } & \multicolumn{2}{|l|}{ Group II } & \multicolumn{2}{|l|}{$t$ test } \\
\hline & Range & Mean \pm SD & Range & Mean \pm SD & $t$ value & $p$ value \\
\hline Total sleep time (h) & $5.2-8.7$ & $6.8 \pm 1$ & $5.9-8.3$ & $6.9 \pm 0.7$ & 0.233 & 0.816 \\
\hline Sleep efficiency (\%) & $68-94$ & $81.2 \pm 5.9$ & $74-92$ & $82.3 \pm 4.7$ & 0.67 & 0.503 \\
\hline Stage 1 latency (min) & $9-22$ & $15.5 \pm 3.4$ & $9-23$ & $15.7 \pm 4.2$ & 0.17 & 0.86 \\
\hline Stage 2 latency (min) & $11-24$ & $17.7 \pm 3.6$ & $12-25$ & $17.8 \pm 4$ & 0.12 & 0.90 \\
\hline Deep sleep latency (min) & $13-26$ & $19.5 \pm 3.6$ & 19-33 & $25.9 \pm 3.7$ & 6.58 & $<0.0001^{*}$ \\
\hline REM latency (min) & 64-105 & $84.7 \pm 11.9$ & $63-102$ & $85.9 \pm 10.4$ & 0.34 & 0.73 \\
\hline N1\% TST & $2-7$ & $4.6 \pm 1.6$ & $3-8$ & $5.5 \pm 1.4$ & 2.26 & $0.027^{*}$ \\
\hline N2\% TST & $11-46$ & $28.7 \pm 11.3$ & $43-57$ & $52.3 \pm 3.5$ & 9.09 & $<0.0001^{*}$ \\
\hline N3\% TST & $25-86$ & $54 \pm 18.4$ & $11-27$ & $20.1 \pm 4$ & 8.13 & $<0.0001^{*}$ \\
\hline REM \% TST & $3-22$ & $12.8 \pm 6$ & $14-28$ & $22.1 \pm 3.8$ & 6.29 & $<0.0001^{*}$ \\
\hline SSTI (/h) & $6.8-30.8$ & $19.5 \pm 6.4$ & $6.7-14.3$ & $9.7 \pm 2.3$ & 6.74 & $<0.0001^{*}$ \\
\hline $\mathrm{AHI}(/ \mathrm{h})$ & $0-3.2$ & $1.4 \pm 0.8$ & $0.2-3.1$ & $1.4 \pm 0.8$ & 0.149 & 0.88 \\
\hline PLMs index (/h) & $2.8-12.6$ & $6.7 \pm 2.6$ & $2.5-11.3$ & $4.77 \pm 0.8$ & 3.24 & $0.002^{*}$ \\
\hline Snore index (/h) & $1.7-60.3$ & $15.2 \pm 15.9$ & $2.9-13.2$ & $6.5 \pm 2.9$ & 2.42 & $0.018^{*}$ \\
\hline
\end{tabular}

AHI apnea hypopnea index, N1,N2, N3 non-rapid eye movement stages 1, 2, 3, PLMs periodic limb movements, REM rapid eye movement, SSTI sleep stage transition index, TST total sleep time

*Significant

Thurber's work (Thurber 2017) who stated the importance of genetic role in pathogenesis of MNE and documented the presence of more than one affected member within the patient's family tree. The present study showed non-significant difference in the incidence of SBO in MNE children and healthy control subjects but enuretic children with SBO had history of poor response to desmopressin. Shin and colleagues (2013) found a nearer incidence of SBO among their studied cases (26.9\%). The study also showed variable response of the included MNE children to previous vasopressin treatment, part had partial response, another was totally non-responsive, and a third group was temporarily responsive which points to the different etiological factors of the disease and the problem is not only disturbed nocturnal vasopressin secretion. This finding is in accordance with Thurber's work (Thurber 2017) who stated that MNE is a multifactorial disorder in which disturbed nocturnal vasopressin secretion constitutes a part but not the whole picture of etiology.

The present study showed a high percent of inverted VP circadian rhythm (52.5\%) and significant decrease in nocturnal/diurnal VP difference in enuretic children than controls pointing to the presence of nocturnal polyuria
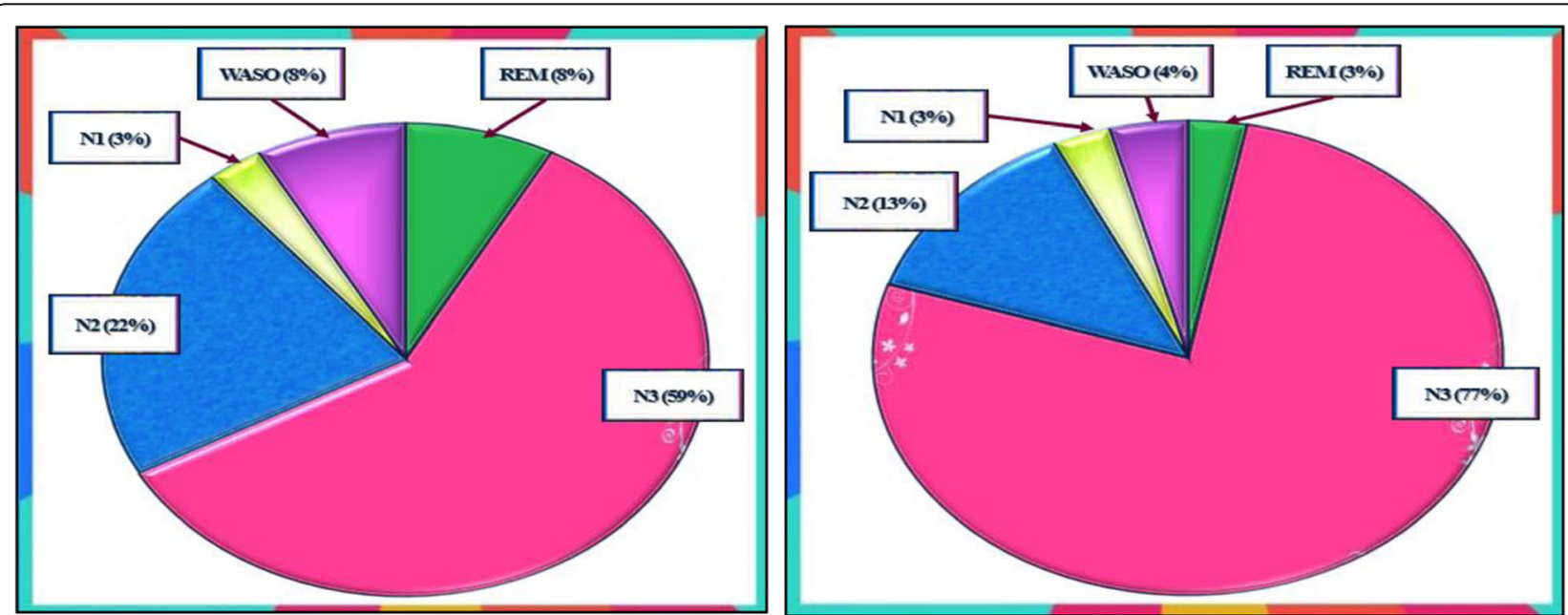

Fig. 1 This figure shows percent of sleep stages from total sleep time in two studied enuretic children with marked prolongation of N3 slow wave deep sleep and shortening of each rapid eye movement sleep (REM) and N2 non-rapid eye movement sleep 


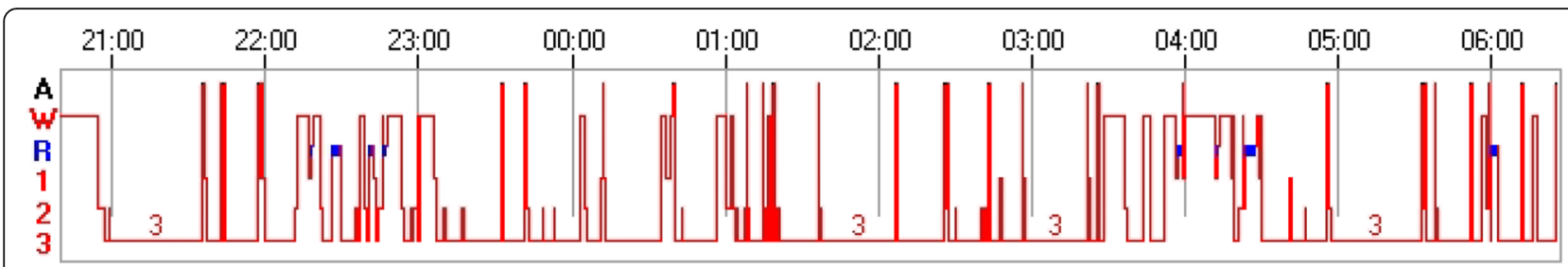

Fig. 2 Hypnogram of a case of nocturnal enuresis showing decreased REM \% of total sleep time and increased each of N3 slow wave deep sleep and sleep stage transition index

among enuretic children. These results are in accordance with Fatouh and colleagues (2013) and Kim and colleagues (2014). At the same time, ElBaz and colleagues (2015) and Hamed and colleagues (2015) are in accordance with that children with inverted rhythm had a higher frequency of enuresis but they estimated a higher percent of inverted vasopressin rhythm among their studied enuretic children (82\%) possibly due to difference in demographic data of their studied children. On the other side, some studies included that of Hoffmann and colleagues (2017) which assumed that decreased nocturnal vasopressin secretion is a consequence and not the cause of enuresis as bladder distension is a potent stimulator of VP secretion and bladder emptying by enuresis may be an inhibitor of VP secretion, but we tried to overcome this debate in the study by unifying the methodology of sample collection in both MNE children and healthy control subjects.

Psychological assessment of enuretic children using CBCL revealed increased anxious depressed symptoms, social problem, attention problems, and internalizing problems in comparison to control subjects. On the other hand, somatic symptoms, thought problems, physical activities, and sluggish cognitive tempo showed no significant differences between children with NE and control subjects. These results are passing with the work of Abd El-Wahab and colleagues (2015) who found impairment of the CBCL competence parameters mainly in participation in activities and social and total competence.

Polysomnographic studies showed marked prolongation in slow wave deep sleep (N3) in enuretic children than controls on the expense of shortening of N1, N2, and REM sleep. The work of Hamed and colleagues (2015) agrees with shortened REM \% of TST among enuretic children but their work showed significant prolongation of N1\% of TST and non-significant differences in each of $\mathrm{N} 2$ and N3\% of TST possibly due to differences in demographic data of their studied subjects. These children may benefit from bedwetting alarm, transcutaneous electrical nerve stimulation of the foot,

Table 3 Correlations between the frequencies of nocturnal enuresis and the nocturnal-diurnal vasopressin difference with other studied parameters among enuretic children group

\begin{tabular}{lllll}
\hline & NE weekly frequency & & \multicolumn{2}{l}{ ND vasopressin difference } \\
\cline { 2 - 3 } & $r$ & 0 & $0.0028^{*}$ & 0.29 \\
\hline Age & -0.46 & $<0.0001^{*}$ & - & 0.07 \\
ND vasopressin difference & -0.86 & 0.69 & 0.05 & - \\
ADS & 0.06 & 0.83 & -0.2 & 0.74 \\
Social problems & 0.03 & 0.83 & 0.075 & 0.86 \\
Attention problems & 0.03 & 0.71 & 0.06 & 0.64 \\
Internalizing problems & 0.06 & 0.36 & 0.06 & 0.71 \\
Deep sleep latency & -0.15 & $<0.0001^{*}$ & 0.75 & 0.70 \\
N1\% TST & -0.71 & $<0.0001^{*}$ & 0.74 & $<0.0001^{*}$ \\
N2\% TST & -0.85 & $<0.0001^{*}$ & -0.80 & $<0.0001^{*}$ \\
N3\% TST & 0.89 & $<0.0001^{*}$ & 0.83 & $<0.0001^{*}$ \\
REM \% TST & -0.92 & $<0.0001^{*}$ & -0.82 & $<0.0001^{*}$ \\
SSTI & 0.87 & 0.07 & -0.22 & $<0.0001^{*}$ \\
PLM index & 0.29 & $<0.0001^{*}$ & -0.87 & 0.16 \\
Snore index & 0.79 & $<0.0001^{*}$ \\
\hline ADS
\end{tabular}

ADS anxious depressed symptoms, N3 non-rapid eye movement stages 3, NE nocturnal enuresis, ND nocturnal diurnal, PLMs periodic limb movements, SSTI sleep stage transition index, TST total sleep time

*Significant 
or further researches on drugs that return the normal sleep architecture.

The study also showed significant increase in SSTI in enuretic children than controls pointing to impaired sleeping quality which in turn may predispose to daytime sleepiness and learning problems. These results are in harmony with that of Soster and colleagues (2016) who found impaired sleep disturbance scale for children in enuretic children. Periodic limb movement and snoring indices were slightly higher in enuretics than control subjects, and these results are passing with those of Dhondt and colleagues (2015) and Alexopoulos and colleagues (2005), respectively.

The frequencies of NE were higher among children with positive family history of NE, inverted VP circadian rhythm, and higher sleep architecture abnormalities. These results are in accordance with the works of Kim and colleagues (2014) and Hamed and colleagues (2015).

\section{Conclusions}

Primary MNE is a multi-etiological disorder results from the interaction of genetic predisposition, sleep architecture abnormalities, inverted vasopressin secretion rhythm, and psychological disturbances. So, its management is in ultimate need of multidisciplinary treatment protocol.

\section{Abbreviations}

AHI: Apnea hypopnea index; CBCL: Child behavior checklist; MNE: Monosymptomatic nocturnal enuresis; N1: Non-rapid eye movement sleep stage 1; N2: Non-rapid eye movement sleep stage 2; N3: Non-rapid eye movement sleep stage 3; NE: Nocturnal enuresis; PLMI: Periodic limb movement index; PSG: Polysomnogram; REM: Rapid eye movement; SBO: Spina bifida occulta; SE: Sleep efficiency; SSTI: Sleep stage transition index; TST: Total sleep time; VP: Vasopressin; WASO: Wake after sleep onset

\section{Availability of data and materials}

The raw data and materials could be publicly available.

\section{Authors' contributions}

WSB participated in the study idea, design, and patients' collection and performed statistical analysis, polysomnographic data analysis, references collection, and manuscript writing and revision. YAH participated in the study design, patients' collection, and study revision. EAE participated in the patients' collection, statistical analysis, manuscript writing, and revision. MAS participated in the study design, psychological assessment of the patients and control, references collection, and manuscript revision. ISI participated in the study design, patient chest evaluation, PSG interpretation, and manuscript revision. AYA participated in the patient and control blood sample collection and evaluation of vasopressin level both diurnal and nocturnal, routine laboratory investigations, and statistical analysis. All authors read and approved the final manuscript.

Ethics approval and consent to participate section

- The manuscript was approved by The Research Ethics Committee and Quality Assurance Unit, Faculty of Medicine, Tanta University.

- The URL: http://tqac.tanta.edu.eg/new-tqac/

QualityAssuranceUnit@hotmail.com

- Approval Code: 30888/04/16

- Name of the PI: Wafik Said Kamel El-Bahnasy

- Name of the department: Neuropsychiatry

- Type of the research: promotion research

- Date of approval: April 2016
- The study protocol was approved by the local ethics committee (The Research Ethics Committee and Quality Assurance Unit, Faculty of Medicine, Tanta University). Participation was voluntary, and all participants' parents received detailed information concerning the aims of this research work and possible risks of participation, and an informed consent was obtained from every case prior to the commencement of the study.

\section{Consent of publication}

All participants' parents had signed an informed consent to participate and for the data to be published.

\section{Competing interests}

There were no financial or non-financial competing interests related to the study

\section{Publisher's Note}

Springer Nature remains neutral with regard to jurisdictional claims in published maps and institutional affiliations.

Received: 4 October 2017 Accepted: 21 June 2018

Published online: 09 July 2018

References

Abd El-Wahab DM, Mubarak AA, El-Sawy HE, Seleem MA. Psychosocial profile of late childhood and adolescence with enuresis. Master thesis. Tanta: Tanta University; 2015. p. 63-4.

Achenbach TM, Dumenci L. Advances in empirically based assessment: revised cross-informant syndromes and new DSM-oriented scales for the CBCL, YSR, and TRF: comment on Lengua, Sadowksi, Friedrich, and Fischer (2001). J Consult Clin Psychol. 2001;69(4):699-702.

Alexopoulos El, Kaditis AG, Kostadima E, Gourgoulianis K. Resolution of nocturnal enuresis in snoring children after treatment with nasal budesonide. Urology. 2005;66:14-6.

Austin PF, Bauer SB, Bower W, Chase J, Franco I, Hoebeke P, et al. The standardization of terminology of lower urinary tract function in children and adolescents: update report from the Standardization Committee of the International Children's Continence Society. J Urol. 2014;191(6):1863-5.

Baeyens D, Roeyers H, Naert S, Hoebeke P, Vande Walle J. The impact of maturation of brainstem inhibition on enuresis: a startle eye blink modification study with 2-year follow-up. J Urol. 2007;178:2621-5.

Berry RB, Brooks R, Gamaldo C, Harding SM, Lloyd RM, Quan SF, et al. AASM Scoring Manual Updates for 2017 (Version 2.4). J Clin Sleep Med. 2017; 13(5): 665-666. https://doi.org/10.5664/jcsm.6576

Butler RJ, Heron J. The prevalence of infrequent bedwetting and nocturnal enuresis in childhood. A large British cohort Scand J Urol Nephrol. 2008;42(3): 257-64.

Dhondt K, Herzeele C, Roels SP, Raes A, Groen L, Hoebeke P, et al. Sleep fragmentation and periodic limb movements in children with monosymptomatic nocturnal enuresis and polyuria. Pediatr Nephrol. 2015;30: 1157-62.

EL Koumi MA, Ali YF, El Banna EA, Youssef UM, Raya YM, Ismail AA. Psychiatric Morbidity among a Sample of Orphanage Children in Cairo. Int J Pediatr. 2012; 2012: 141854.

EIBaz F, Zahra S, Khairy M. Assessment of the efficacy of desmopressin in treatment of primary monosymptomatic nocturnal enuresis in Egyptian children. Egyptian J Med Human Gen. 2015;16:257-62.

Fatouh AA, Motawie AA, Abd Al-Aziz AM, Hamed HM, Awad MA, Abd El-Ghany $A$, et al. Anti-diuretic hormone and genetic study in primary nocturnal enuresis. J Pediatr Urol. 2013;9:831-7.

Hamed HM, Abd Al-Aziz AM, Motawie AA, Fatouh AA, Mona Awad MA, Afifi L. Polysomnography and antidiuretic hormone secretion pattern in children with primary nocturnal enuresis. Egyptian J Neuro Psychiatry Neuro. 2015; 52(4):258-63.

Hoffmann A, Sampaio C, Nascimento AA, Veiga ML, Barroso U. Predictors of outcome in children and adolescents with overactive bladder treated with parasacral transcutaneous electrical nerve stimulation. J Pediatr Urol. 2017; (17):30313-3. https://doi.org/10.1016/j.jpurol.2017.07.017.

Joinson C, Heron J, Emond A, Butler R. Psychological problems in children with bedwetting and combined (day and night) wetting: a UK population-based study. J Pediatr Psychol. 2007;32(5):605-16. 
Khedr EM, Abo-Elfetoh N, Elbeh KA, Baky AA, Gamal RM, El Hammady D, et al. Transcranial magnetic stimulation identifies cortical excitability changes in monosymptomatic nocturnal enuresis. Neurophysiologie Clinique/Clinical Neurophysiology. 2015;45:151-8.

Kim JM, Park JW, Lee CH. Evaluation of nocturnal bladder capacity and nocturnal urine volume in nocturnal enuresis. J Pediatr Urol. 2014;10:559-63.

Mota DM, Victora CG, Hallal PC. Investigation of voiding dysfunction in a population-based sample of children aged 3 to 9 years. J Pediatr. 2005; 81:225-32.

Raheem AA, Farahat Y, El-Gamal O, Ragab M, Radwan M, El-Bahnasy AH, et al. Role of posterior tibial nerve stimulation in the treatment of refractory monosymptomatic nocturnal enuresis: a pilot study. J Urol. 2013;189:1514-8.

Shin Sh IYJ, Lee MJ, Lee YS, Choi EK, Han SW. Spina bifida occulta: not to be overlooked in children with nocturnal enuresis. Int J Urol. 2013;20(8):831-5.

Soster LA, Alves R, Fagundes SN, Koch VH, Bruni O. Sleep disturbances associated with sleep enuresis: a questionnaire study. Europ J Paediatric Neurology. 2016:20:282-5.

Telli O, Samancı C, Sarıc H, Hascıcek AM, Kabar M, Eroglu M. Can urinary nerve growth factor and bladder wall thickness correlation in children have a potential role to predict the outcome of non-monosymptomatic nocturnal enuresis? J Pediatr Urol 2015; 11:265. e1-5. DOl: https://doi.org/10.1016/j. jpurol.2015.03.018

Thurber S. Childhood enuresis: current diagnostic formulations, salient findings, and effective treatment modalities. Arch Psychiatr Nurs. 2017;31(3):319-23. https://doi.org/10.1016/j.apnu.2016.11.005.

von Gontard A, Heron J, Joinson C. Family history of nocturnal enuresis and urinary incontinence: results from a large epidemiological study. J Urol. 2011; 185:2303-7. https://doi.org/10.1016/j.juro.2011.02.040.

\section{Submit your manuscript to a SpringerOpen ${ }^{\circ}$ journal and benefit from:}

- Convenient online submission

- Rigorous peer review

- Open access: articles freely available online

- High visibility within the field

- Retaining the copyright to your article

Submit your next manuscript at $\gg$ springeropen.com 